\title{
Energy-Conserving Simulation of Incompressible Electro-Osmotic and Pressure-Driven Flow
}

\author{
Jahrul Alam \\ Department of Mathematics \\ Shah Jalal University of Science and Technology \\ Sylhet, Bangladesh \\ John C. Bowman ${ }^{1}$ \\ Department of Mathematical Sciences \\ University of Alberta \\ Edmonton, AB T6G 2G1 \\ Canada \\ (accepted May 10, 2002 in Theoretical and Computational Fluid Dynamics)

\begin{abstract}
A numerical model for electro-osmotic flow is described. The advecting velocity field is computed by solving the incompressible Navier-Stokes equation. The method uses a semi-implicit multigrid algorithm to compute the divergence-free velocity at each grid point. The finite differences are second-order accurate and centered in space; however, the traditional second-order compact finite differencing of the Poisson equation for the pressure field is shown not to conserve energy in the inviscid limit. We have designed a non-compact finite differencing for the Laplacian in the pressure equation that allows exact energy conservation and affords second-order accuracy. The model also incorporates a new numerical method for passive scalar advection, called parcel advection, which accurately predicts the evolution of a passively traveling scalar pulse without requiring the addition of any artificial diffusion. The algorithm is used to confirm the experimentally observed asymmetric concentration profile that arises when an external pressure drop is imposed on electro-osmotic flow.
\end{abstract}

\section{Introduction}

Extensive experimental studies of the application of an electric field to a mixture of charged species in solution have been performed in microfluidic experiments and in chemical analyses of biological fluids (Jorgenson \& Lukacs, 1981; Culbertson \& Jorgenson, 1994; Seiler et al., 1994; Culbertson et al., 1998)— for example, for protein separation and DNA sequencing. In laboratory experiments, the solvent flow can be directed along a specified microchannel by the application of appropriate voltages (Harrison et al., 1992). In most of these experiments, the sample was injected with an electro-osmotic flow driven by an applied potential along the channel (Harrison et al., 1993; Seiler et al., 1994). In the field of analytical sciences, particularly in the area of DNA diagnostics or protein separation, the technique of electrokinetic injection has advanced very rapidly during the last few decades. The fundamental principles of microflow injection are based on the double-layer theory developed by von Helmholtz (1879) and the linearized ion energy

\footnotetext{
^Email: bowman@math.ualberta.ca
} 
distribution of Debye \& Hückel (1923). The fact that the application of an electric field can induce a fluid flow through a microchannel was observed as far back as the beginning of the 19th century (Ruess, 1809). Microfluidic devices composed of a network of channels are being used in the chemical and biochemical industries in order to reduce both the time and cost of analytical procedures. Computer simulations can be used along with empirical studies to investigate the efficiency of such microfluidic devices.

The phenomena that we deal with throughout this study are the effects associated with the distribution of charged substances that are dissolved in a fluid near an interface with constant electric charge (Morrison \& Stukel, 1970). They play an important role in many diverse natural and technological processes, such as in the biotechnology industry and in biochemical and biological research. When a charged interface is brought in contact with an ionized fluid, the electrical charges on the interface will attract ions in the fluid that have opposite sign; hence there is a layer of fluid near the charged interface where the counter-ion concentration is much higher than the co-ion concentration (Arulanandam \& Li, 2000). In other words, there is a layer, known as the Debye sheath, next to the charged interface, which has a net electrical charge concentrated in a small volume. The Debye-Hückel approximation (Debye \& Hückel, 1923) can be used to show that the thickness $\lambda_{D}$ of this layer depends on the ion density $n$ of the fluid and is given by $\lambda_{D}^{2}=k T \epsilon /\left(e^{2} n\right)$, where $k$ is the Boltzmann constant, $T$ is the absolute temperature, $e$ is the elementary charge, and $\epsilon$ is the electrical permittivity of the fluid. Typical experimental values of $\lambda_{D}$ lie between $1 \mathrm{~nm}$ and $100 \mathrm{~nm}$. When an electrical field is applied to the liquid, the excess counter-ions in the Debye layer will move under the applied electric field. These ions will migrate to the electrode that has opposite sign.

We consider an electro-osmotic device consisting of a channel with a rectangular cross section filled with a buffering medium across which a voltage is applied. A schematic diagram of the electric double layer that develops at the charged interface is shown in Figure 1. Suppose that the wall of the channel is negatively charged and a positive voltage is applied at the input reservoir on one end of the channel. Since the interface is negatively charged, the positive charges in the solution are concentrated near the wall. These charges will move toward the negative electrode (Reed \& Morrison, 1976), interact frictionally with the surrounding fluid, pulling it with them and generating a fluid flow. This is the so-called electro-osmotic flow. The bulk behavior of a solution of charged particles is complicated by the dynamics of the diffuse space charge that arises from the response of the ions to the charged interface (Levine \& Neale, 1976). However, if this complication is ignored, the dynamics of an ionic solution near a charged interface can be understood from a description of the electric fields and forces operating in the fluid (Ermakov et al., 2000). The overall dynamics can be modeled mathematically using the conservation of mass and momentum, together with the theory of Debye and Hückel.

Chemical separation procedures of various species, including the separation of small ions in solution, have been investigated both theoretically and experimentally. As well, mathematical models and computeraided tools have been developed. The details of the mathematical models can be found in Babskii et al. (1983, and refs. therein) and in Mosher et al. (1992, and refs. therein). Because these mathematical models consist of nonlinear partial differential equations, an exact analytical solution of a model cannot normally be obtained for arbitrary initial and boundary conditions unless the model is linearized. Numerical computation can provide approximate solutions to the fully nonlinear model. Jorgenson \& Lukacs (1981) developed a one-dimensional model of capillary electrophoresis. These authors demonstrated electrophoretic separation efficiency of chemical and biological substances in open-tubular glass capillaries of $75 \mu \mathrm{m}$ inside diameter. An analytical attempt to investigate electro-osmotic flow in a single long uniform capillary can be found in Rice \& Whitehead (1965), Burgreen \& Nakache (1964), and Andreev \& Lisin (1993). Two-dimensional computer simulations of electrokinetic injection and transport phenomena in a cross-channel device (see Figure 2) are described in Ermakov et al. (1998, 2000). A steady-state three-dimensional simulation was performed by Patankar \& Hu (1998) to study the electro-osmotic flow in a cross-channel microfluidic device. Fletcher et al. (1999) described electro-osmotic flows and electrophoretic separation procedures and solved the one-dimensional electrophoresis equation numerically using a Crank-Nicholson scheme, where the chemical reaction terms were resolved. They found that the numerical scheme loses accuracy if the reaction is much faster than the diffusion process and proposed a fully implicit method. Major numerical difficulties associated with electro-osmosis and electrophoresis are well known to numerical analysts in this area. Particularly important issues in simulating microchannel flows are (i) complicated computational geometries, (ii) the solenoidal nature of the velocity field, (iii) the formation of shock and 


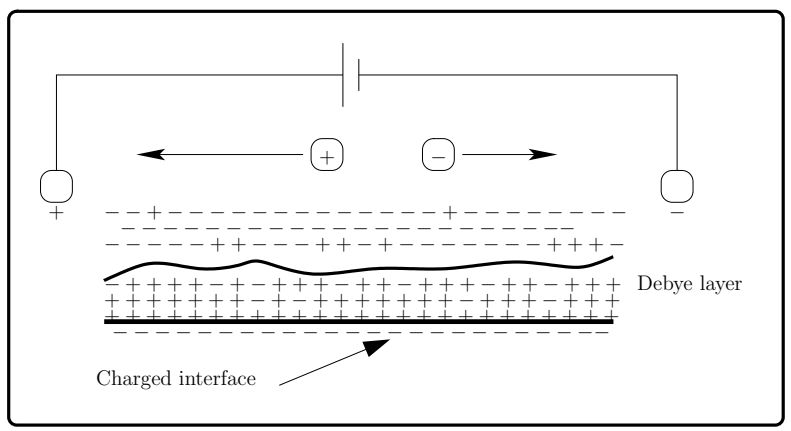

Figure 1. A schematic diagram of the electric double layer that develops at the charged interface.

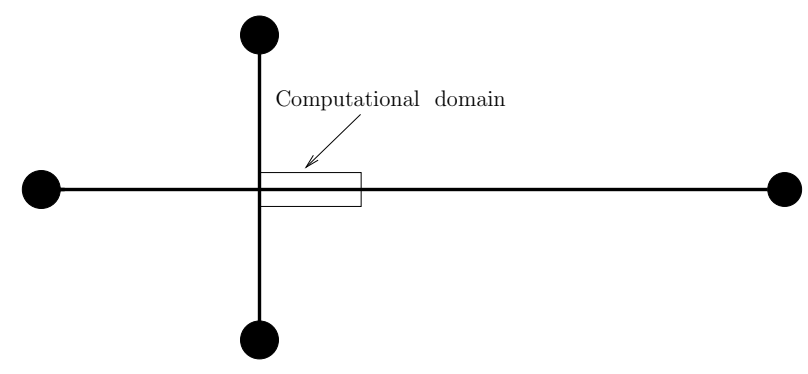

Figure 2. A cross-channel microfluidic device.

contact discontinuities because of the slowly diffusing nature of the chemical species, and (iv) resolving the Debye layer in the numerical grid. We have ignored the first issue by restricting our simulation to a simple geometry. Since the typical value of the Debye layer thickness is in the nanometer range, Patankar \& Hu (1998) and others have adopted higher values of this parameter and/or used a finer grid near the Debye region. It has been demonstrated analytically (Rice \& Whitehead, 1965) and numerically (e.g., see Alam (2000)) that increasing the non-dimensional value of the Debye layer thickness may change the gross characteristics of the electro-osmotic flow. However, at a sufficiently low ion concentration, the thickness of the Debye layer is large enough to be readily modeled with a uniform grid.

Electro-osmosis is associated with substances that diffuse very slowly when dissolved in a buffering medium. Experiments show that the typical value of the diffusion coefficient of the concentration field is in the range of $10^{-12} \mathrm{~m}^{2} / \mathrm{s}$ to $10^{-8} \mathrm{~m}^{2} / \mathrm{s}$. Thus the dissolved sample primarily experiences advection by the fluid and electrophoretic velocities. Eulerian schemes for advection are unconditionally unstable and can produce spurious wiggles and even negative values of the concentration. We have therefore developed a fully Lagrangian algorithm to simulate passive advection (Bowman et al., 2002). We have also developed a numerical algorithm to simulate two-dimensional incompressible electro-osmotic flow such that the solenoidal nature of the velocity field is preserved and, in the inviscid and unforced limit, the kinetic energy of the fluid is exactly conserved.

One of the primary aims of this study is to develop and optimize numerical techniques to study electroosmosis and electrophoresis. For simplicity, we restrict the computational domain to a two-dimensional rectangular channel. While the restriction to two dimensions is thought to be a good approximation to describe passive transport by electro-osmotic flow (Patankar \& Hu, 1998), our numerical code has been designed so that it can be readily generalized to three dimensions.

In the following sections we describe the fundamental partial differential equations that model the phenomena of electro-osmosis and electrophoresis, the numerical algorithm that preserves the solenoidal nature of the flow field, and some results of our numerical simulations. 


\section{Governing Equations}

We assume that the buffer inside the channel is a fluid that is incompressible and Newtonian with uniform physical properties, the thickness of the Debye layer is small compared with the characteristic length scale, and the concentration of the dissolved species is dilute and does not affect other physical properties of the fluid such as its density. This assumption does not necessarily require that the density of the fluid be constant; it only requires that the density be independent of the concentration field. In other words, the concentration field is a passive scalar quantity (e.g., see Leslie (1973)).

The fluid density $\rho(\boldsymbol{x}, t)$ and fluid velocity $\boldsymbol{v}(\boldsymbol{x}, t)$ satisfy the equations for incompressibility,

$$
\frac{\partial \rho}{\partial t}+\boldsymbol{v} \cdot \nabla \rho=0
$$

and conservation of mass,

$$
\frac{\partial \rho}{\partial t}+\nabla \cdot(\boldsymbol{v} \rho)=0
$$

These two equations can be combined together to yield the solenoidal condition

$$
\boldsymbol{\nabla} \cdot \boldsymbol{v}=0
$$

regardless of whether the density is uniform or not.

The buffer can often be assumed to be of uniform density, so for the rest of this study we assume that $\rho$ is constant everywhere in the domain. The flow field will then satisfy

$$
\frac{\partial \boldsymbol{v}}{\partial t}+(\boldsymbol{v} \cdot \boldsymbol{\nabla}) \boldsymbol{v}=-\frac{1}{\rho} \nabla P+\nu \nabla^{2} \boldsymbol{v}+\boldsymbol{F}
$$

where $P(\boldsymbol{x}, t)$ is the pressure, $\nu$ is the kinematic viscosity of the fluid, and $\boldsymbol{F}(\boldsymbol{x}, t)$ is the electric force per unit mass. The concentration $C(\boldsymbol{x}, t)$ of the species dissolved in the buffer will then satisfy

$$
\frac{\partial C}{\partial t}+\nabla \cdot \Gamma=D \nabla^{2} C
$$

where $\Gamma$ is the flux of $C$ per unit volume and $D$ is the diffusion coefficient. The concentration flux $\Gamma$ is given by (Saville, 1977)

$$
\boldsymbol{\Gamma}=(\boldsymbol{v}+\mu \boldsymbol{E}) C,
$$

where $\mu$ is the electrophoretic mobility (assumed to be a constant, proportional to the sign of the elementary charge $q_{e}$ of the species), and $\boldsymbol{E}$ is the electric field intensity. Note that $\boldsymbol{v} C$ is the electro-osmotic flux, due to the bulk fluid flow, and $\mu \boldsymbol{E C}$ is the flux due to electrophoresis.

Thus we have two partial differential equations, (4) and (5), to be solved subject to the constraint (3), for the vector $\boldsymbol{v}$ and two scalars $P$ and $C$. The body force $\boldsymbol{F}$ is calculated using the electrical properties of the fluid. The advected species is dilute, so that the fluid properties are independent of the concentration field. The concentration field thus does not appear in the momentum balance; that is, (4) is decoupled from (5). Once the velocity field has been computed from (3) and (4), coupled with the equations for the electrical field, the concentration distribution can then be determined from (5).

\subsection{Pressure Field}

The solution of this system of partial differential equations is complicated by the absence of a dynamic equation for the pressure field. The pressure field is determined in such a way that a divergence-free velocity field is achieved. On taking the divergence of (4),

$$
\boldsymbol{\nabla} \cdot\left(\frac{\partial \boldsymbol{v}}{\partial t}+\boldsymbol{v} \cdot \boldsymbol{\nabla} \boldsymbol{v}\right)=\nabla \cdot\left(-\frac{1}{\rho} \nabla P+\nu \nabla^{2} \boldsymbol{v}+\boldsymbol{F}\right)
$$

and using (3), we obtain a Poisson equation that can be solved for $P$,

$$
\nabla^{2} P=\rho \nabla \cdot(\boldsymbol{F}-\boldsymbol{v} \cdot \boldsymbol{\nabla} \boldsymbol{v})
$$


Equation (8) is an elliptic partial differential equation that can be solved numerically for $P$ using an iterative technique, such as a multigrid Poisson solver (e.g., see Bowman et al. (2000)). The velocity and the pressure fields are related in such a way that the pressure field will self-consistently adjust to maintain the solenoidal nature of the velocity field. In a numerical computation, the velocity field should be computed such that (3) is satisfied at each time step. Although no time derivative appears in (8), it has to be solved at each time step to find the pressure distribution required to maintain the solenoidal nature of the velocity field.

\subsection{Body Force}

The body force $\boldsymbol{F}$ in (4) can be related to the electric field intensity $\boldsymbol{E}$ and the space-charge density $\rho_{e}=q_{e} C$, where $q_{e}$ is the elementary charge of the species, by

$$
\rho \boldsymbol{F}=\rho_{e} \boldsymbol{E},
$$

where $\boldsymbol{E}$ is related to the total potential $\Phi$ via $\boldsymbol{E}=-\nabla \Phi$. Using Maxwell's equations, one obtains a Poisson equation for the potential,

$$
\nabla^{2} \Phi=-\frac{\rho_{e}}{\epsilon} .
$$

The potential field $\Phi$ thus arises due to the space charge density $\rho_{e}$. In the case of electro-osmosis, the total potential $\Phi$ has a contribution from the externally applied electric field and another contribution due to the charge at the walls and in the interior of the computational domain. It is thus convenient to decompose $\Phi=\varphi+\psi$, where $\varphi$ is the potential due to the externally applied electric field and $\psi$ is the potential due to the space charges in the computational domain. Since the applied potential arises only from external charges, it satisfies Laplace's equation within the domain,

$$
\nabla^{2} \varphi=0 .
$$

Using (10) and (11), it follows that the potential $\psi$ also satisfies Poisson's equation, $\nabla^{2} \psi=-\rho_{e} / \epsilon$, relating $\psi$ to $\rho_{e}$. The charge distribution in the solution is governed by the potential at the interface. The Debye-Hückel approximation leads to the Helmholtz equation

$$
\nabla^{2} \psi=\frac{1}{\lambda_{D}^{2}} \psi
$$

Equation (9) then yields $\boldsymbol{F}=\epsilon \psi \boldsymbol{\nabla}(\varphi+\psi) /\left(\rho \lambda_{D}^{2}\right)$. The concentration flux $\boldsymbol{\Gamma}$ given by (6) can also be written in terms of the potential fields as $\boldsymbol{\Gamma}=[\boldsymbol{v}-\mu \boldsymbol{\nabla}(\varphi+\psi)] C$.

One can now write down the model equations in a closed solvable form, as described in the following subsection.

\subsection{Model Equations}

Using a tilde to denote dimensional quantities, and using the fact that away from the wall the derivatives of $\psi$ are negligible compared with those of $\varphi$, the governing equations can be written as

$$
\begin{gathered}
\frac{\partial \tilde{\boldsymbol{v}}}{\partial \tilde{t}}+\tilde{\boldsymbol{v}} \cdot \tilde{\nabla} \tilde{\boldsymbol{v}}=-\frac{1}{\rho} \tilde{\boldsymbol{\nabla}} \tilde{P}+\tilde{\nu} \tilde{\nabla}^{2} \tilde{\boldsymbol{v}}+\frac{\epsilon}{\rho \lambda_{D}^{2}} \tilde{\psi} \tilde{\boldsymbol{\nabla}} \tilde{\varphi} \\
\frac{\partial \tilde{C}}{\partial \tilde{t}}+\tilde{\boldsymbol{\nabla}} \cdot(\tilde{\boldsymbol{v}}-\tilde{\mu} \tilde{\boldsymbol{\nabla}} \tilde{\varphi}) \tilde{C}=\tilde{D} \tilde{\nabla}^{2} \tilde{C} \\
\tilde{\nabla}^{2} \tilde{P}=\rho \tilde{\nabla} \cdot\left(\frac{\epsilon}{\rho \lambda_{D}^{2}} \tilde{\psi} \tilde{\boldsymbol{\nabla}} \tilde{\varphi}-\tilde{\boldsymbol{v}} \cdot \tilde{\nabla} \tilde{\boldsymbol{v}}\right), \\
\tilde{\nabla}^{2} \tilde{\psi}=\frac{1}{\lambda_{D}^{2}} \tilde{\psi} \\
\tilde{\nabla}^{2} \tilde{\varphi}=0 .
\end{gathered}
$$


These equations form a closed system that can be non-dimensionalized by defining a characteristic length scale and a characteristic velocity scale. For electro-osmotic flow associated with micro- and nano-technology, the typical length scales are $10^{-6} \mathrm{~m}$ and $10^{-9} \mathrm{~m}$, respectively. Since the flow characteristics in electro-osmotic pumping are dynamically similar to a channel flow, we chose the width $h$ of the device used in the laboratory experiments as our characteristic length scale. A typical velocity $U$ of the fluid due to electro-osmosis is taken as a characteristic velocity scale. Accordingly, we introduce the non-dimensional variables

$$
\boldsymbol{x}=\frac{\tilde{x}}{h}, \boldsymbol{v}=\frac{\tilde{\boldsymbol{v}}}{U}, t=\frac{U \tilde{t}}{h}, P=\frac{\tilde{P}}{\rho U^{2}}, \varphi=\frac{\tilde{\varphi}}{\Phi_{*}}, \psi=\frac{\tilde{\psi}}{\Phi_{*}} .
$$

Here the pressure is scaled by $\rho U^{2}$ and a typical value $\Phi_{*}$ is used to normalize the external and internal potentials.

The governing equations can now be written in a flux-conservative form using the solenoidal nature of the velocity field. In dimensionless variables, the equations take the form

$$
\begin{gathered}
\frac{\partial \boldsymbol{v}}{\partial t}+\boldsymbol{\nabla} \cdot(\boldsymbol{v} \boldsymbol{v})=-\boldsymbol{\nabla} P+\nu_{v} \nabla^{2} \boldsymbol{v}+\alpha \psi \boldsymbol{\nabla} \varphi \\
\frac{\partial C}{\partial t}+\boldsymbol{\nabla} \cdot(\boldsymbol{v}-\mu \boldsymbol{\nabla} \varphi) C=\nu_{c} \nabla^{2} C \\
\nabla^{2} P=\boldsymbol{\nabla} \cdot(\alpha \psi \boldsymbol{\nabla} \varphi-\boldsymbol{v} \cdot \boldsymbol{\nabla} \boldsymbol{v}) \\
\nabla^{2} \psi=\kappa^{2} \psi \\
\nabla^{2} \varphi=0
\end{gathered}
$$

where

$$
\alpha=\frac{\Phi_{*}^{2} \epsilon}{\rho U^{2} \lambda_{D}^{2}}=\frac{\Phi_{*}^{2} e^{2} n}{\rho U^{2} k T}, \nu_{v}=\frac{\tilde{\nu}}{U h}, \nu_{c}=\frac{\tilde{D}}{U h}, \mu=\frac{\tilde{\mu} \Phi_{*}}{U h}, \kappa^{2}=\frac{h^{2}}{\lambda_{D}^{2}}=\frac{h^{2} e^{2} n}{k T \epsilon} .
$$

The values of the dimensionless constants here determine the underlying physics and nature of the flow field. In order to realize the relative significance of the inertial force over the viscous force, we can use typical values of the dimensional quantities to estimate $\nu_{v}$ and $\nu_{c}$, as listed in Table 1 (Harrison et al., 1992; Crabtree et al., 2001). Using these typical values, we find that $\nu_{v} \approx 20$ and $\nu_{c} \sim 10^{-3}$. Hence the velocity field is dominated by viscosity and the concentration field is dominated by advection. However, the diffusion term cannot be neglected from (20) when the duration of an electrophoretic experiment is comparable with the diffusion time scale. The smallness of the non-dimensional number $\nu_{c}$ indicates only that diffusion is slower than advection. Equations (19)-(23) need to be solved for one vector and four scalar quantities. With suitable initial and boundary conditions, they constitute a closed system. The fact that (22) and (23) are time independent can be exploited in a numerical implementation of this system of equations.

\section{Geometry and Boundary Conditions}

\subsection{Computational Geometry}

In this work we restrict our attention to two-dimensional flow in a rectangular domain $\Omega=\left\{(x, y): x_{\min } \leq\right.$ $\left.x \leq x_{\max }, y_{\min } \leq y \leq y_{\max }\right\}$ that represents a horizontal cross section of a single long capillary, as illustrated in Figure 3. The domain has an input boundary at $x=x_{\min }$, an output boundary at $x=x_{\max }$, and two wall boundaries at $y=y_{\min }$ and $y=y_{\max }$. In a numerical simulation, the boundary conditions must be consistent with one another to avoid numerical instability. The behavior of the field variables at the boundary of the computational domain determines the corresponding boundary conditions.

\subsection{Boundary Conditions}

We now describe the boundary conditions used for different fields $\varphi, \psi, P, \boldsymbol{v}, C$, and $\Gamma$. For the external potential field $\varphi$, a Dirichlet boundary condition at the input and output boundaries and a Neumann boundary 


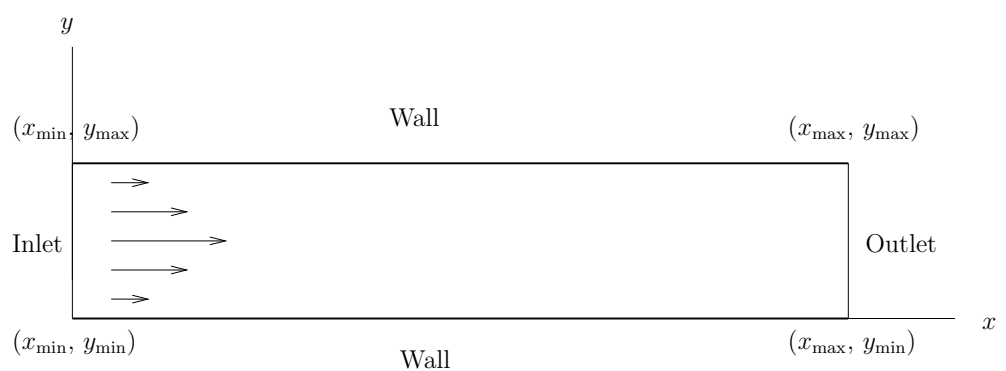

Figure 3. Computational domain representing the horizontal cross section of an electrophoretic microchannel.

condition at the walls (Levine \& Neale, 1976) are used. We use the non-dimensional value $\varphi_{0}$ at the input boundary and $\varphi_{1}$ at the output boundary for $\varphi$ :

$$
\varphi\left(x_{\min }, y\right)=\varphi_{0}, \quad \varphi\left(x_{\max }, y\right)=\varphi_{1}, \quad \frac{\partial \varphi}{\partial y}\left(x, y_{\min }\right)=0, \quad \frac{\partial \varphi}{\partial y}\left(x, y_{\max }\right)=0 .
$$

For the potential due to the charges in the fluid, we use a Dirichlet boundary condition on the walls. To model a solid-liquid interface that has a negative charge, we use the non-dimensional value $-\zeta$ for $\psi$ at the walls. We use a Neumann boundary condition for $\psi$ at the inlet and outlet boundaries:

$$
\frac{\partial \psi}{\partial x}\left(x_{\min }, y\right)=0, \quad \frac{\partial \psi}{\partial x}\left(x_{\max }, y\right)=0, \quad \psi\left(x, y_{\min }\right)=-\zeta, \quad \psi\left(x, y_{\max }\right)=-\zeta .
$$

Using a Dirichlet boundary condition, we specify the dimensionless pressure values at the input and output boundaries. A Neumann boundary condition is used for the pressure at the walls. Thus, the boundary conditions on $P$ are similar to those used by Sidilkover \& Ascher (1995):

$$
P\left(x_{\min }, y\right)=P_{0}, \quad P\left(x_{\max }, y\right)=P_{1}, \quad \frac{\partial P}{\partial y}\left(x, y_{\min }\right)=0 \quad \text { and } \quad \frac{\partial P}{\partial y}\left(x, y_{\max }\right)=0,
$$

where $P_{0}$ and $P_{1}$ are the non-dimensional pressure values at the input and output boundaries, respectively. For the velocity field, no-slip boundary condition on the walls are used. Assuming that the channel is very long, we model only a small section of its length and adopt Neumann boundary conditions at the input and output boundaries,

$$
\frac{\partial \boldsymbol{v}}{\partial x}\left(x_{\min }, y\right)=0, \quad \frac{\partial \boldsymbol{v}}{\partial x}\left(x_{\max }, y\right)=0, \quad \boldsymbol{v}\left(x, y_{\min }\right)=0 \quad \text { and } \quad \boldsymbol{v}\left(x, y_{\max }\right)=0 .
$$

For the concentration field, we adopt a Neumann boundary condition on the walls and a Dirichlet boundary condition at the input and output:

$$
C\left(x_{\min }, y\right)=C_{0}, \quad C\left(x_{\max }, y\right)=C_{1}, \quad \frac{\partial C}{\partial y}\left(x, y_{\min }\right)=0 \quad \text { and } \quad \frac{\partial C}{\partial y}\left(x, y_{\max }\right)=0,
$$

where $C_{0}$ and $C_{1}$ are the concentrations in the input and output reservoirs, respectively. It is also necessary to impose an additional boundary condition for the fluxes in order to finite difference the momentum and concentration equations. A Neumann boundary condition is used on $\Gamma_{x}$ at the inlet and outlet boundaries and a Dirichlet condition is used on $\Gamma_{y}$ at the walls:

$$
\frac{\partial \Gamma_{x}}{\partial x}\left(x_{\min }, y\right)=\frac{\partial \Gamma_{x}}{\partial x}\left(x_{\max }, y\right)=0, \quad \Gamma_{y}\left(x, y_{\min }\right)=\Gamma_{y}\left(x, y_{\max }\right)=0,
$$

where

$$
\boldsymbol{\Gamma}=\left[\begin{array}{l}
\Gamma_{x} \\
\Gamma_{y}
\end{array}\right]
$$

denotes the flux of a field variable. 


\section{Conservation Laws}

In order to study the underlying physics of electro-osmotic flow, we presented in Section 2.3 the fully nonlinear set of partial differential equations. However, to understand the role of the nonlinearity in driving the evolution toward a steady state, it is useful to consider the non-dissipative limit of the system. For an inviscid flow in the absence of an applied pressure drop, electromotive forcing, and diffusion of the scalar field $C$, the energy $E=\frac{1}{2} \int v^{2} d \mathbf{x}$ (mean-squared velocity), enstrophy $Z=\frac{1}{2} \int \omega^{2} d \mathbf{x}$, (meansquared vorticity), mean-squared concentration $\mathcal{C}=\frac{1}{2} \int C^{2} d \mathbf{x}$, and cross correlation $I=\frac{1}{2} \int C \omega d \mathbf{x}$ are all conserved. Here the scalar $\omega$ is the magnitude of the vorticity $\boldsymbol{\omega}=\boldsymbol{\nabla} \times \boldsymbol{v}$. While the energy $E$ and the mean-squared concentration $\mathcal{C}$ are conserved both in two and three dimensions, the enstrophy $Z$ and the cross-correlation $I$ are conserved only in two dimensions. The energy is conserved if there is no viscosity or forcing and the field $\mathcal{C}$ is conserved in the absence of diffusion. Note that in the presence of fluid viscosity and body forces, the energy and enstrophy are not conserved, but, to the extent that scalar diffusion can be neglected, the mean-squared concentration $\mathcal{C}$ remains invariant.

\section{Computational Procedure}

In order to integrate the governing partial differential equations, we have developed a semi-implicit multigrid algorithm to compute the electro-osmotic velocity field such that energy is conserved in the inviscid unforced limit. The concentration field is computed using parcel advection, a new numerical method for fluid simulations. In order to implement the algorithm, a $\mathrm{C}^{++}$module has been developed for the object-oriented initial-value code TRIAD, which provides general facilities for parameter input, parsing, generic integration algorithms, dynamic time stepping, and a restart facility. All the spatial derivatives are calculated using a second-order accurate centered-in-space finite-difference formula. The code TRIAD is designed to solve a system of first-order time-dependent ordinary differential equations.

\subsection{Discretization}

The partial differential equations that model fluid flow can be discretized using the finite-difference method. In the area of computational fluid dynamics there have been two basic approaches to applying the finitedifference method to the governing partial differential equations. One is the Eulerian approach and the other is the Lagrangian approach. One of the most severe drawbacks of the Eulerian method is the difficulty found in dealing correctly with the advective term $\boldsymbol{v} \cdot \boldsymbol{\nabla} \boldsymbol{v}$. In the case of scalar advection of a tracer field, the concentration must always remain non-negative. Without proper handling of advection terms, numerical solutions can go unconditionally unstable, producing negative concentrations or spurious wiggles (Tannehill et al., 1997; Press et al., 1997). Historically, the first alternative was the unconditionally stable method of characteristics introduced by Courant et al. (1952) (sometimes referred to as the CIR scheme; see LeVeque (1990)). Since that method uses interpolation based on the nearest grid point, in spite of its unconditional stability, the overall performance is limited by the inherent computational damping associated with interpolation (Ritchie, 1986).

For the concentration field, we employ an operator-splitting scheme, where different numerical discretizations are used for the diffusion and advection terms. The dynamics of the concentration field are dominated by the advective derivative since the typical value of the dimensionless diffusion coefficient is much smaller than one. Although a wide variety of finite-difference approaches can be used to simulate an advection problem on an Eulerian grid, all of them must respect a Courant-Friedrich-Lewy criterion to remain stable (see e.g., Press et al. (1997)). A fully Lagrangian advection algorithm (Bowman et al., 2002), combined with a Crank-Nicholson scheme for the diffusive part, has been applied to evolve the concentration field. The discretization involves a numerical grid consisting of a finite number of cells. Each of the field variables are computed at the centroid or grid point of each cell. The boundary of the computational domain passes through the centroid of the boundary cells.

Let $n_{x}$ and $n_{y}$ be the total number of cells in the $x$ and $y$ directions respectively and let $(i, j)$ be the location of a cell centroid, where $i=1, \ldots, n_{x}$ and $j=1, \ldots, n_{y}$. The cells located at $(1, j),\left(n_{x}, j\right),(i, 1)$, 
and $\left(i, n_{y}\right)$ for any $i$ or $j$ are boundary cells that are used to implement boundary conditions; for example, a Dirichlet boundary condition requires fixed values at these boundary cells. In order to implement a Neumann boundary condition, we need additional cells around the domain known as ghost cells; they are located at $(0, j),\left(n_{x}+1, j\right),(i, 0)$, and $\left(i, n_{y}+1\right)$. The fluid occupies the whole domain and is distributed over the grid cells. The fluid that occupies one grid cell is called a parcel of fluid. In analogy to the particle-in-cell method, at each time step, a parcel is assigned a new velocity, pressure, and concentration equal to the area-weighted average of these quantities in the cells it overlaps. Initially, the cells and parcels are coincident. As the fluid evolves, the shape of the parcels will in general become distorted; however, because the laminar flows in this work are virtually uniform, rigid advection of the parcels was found to describe the evolution of the concentration field sufficiently accurately.

In the momentum equation, the viscous force dominates the inertial force. The nonlinearity in the advective term can thus be treated explicitly on the right-hand side as a source term. A Crank-Nicholson scheme, which is unconditionally stable (Richtmyer \& Morton, 1957), works well for the viscous term. However, there are particular difficulties in dealing with the pressure term. Specifically, one should compute the pressure in such a way that the incompressibility of the velocity field is maintained.

For the purpose of discretizing the governing equations, we define the discrete operator

$$
\boldsymbol{\nabla}_{1} F_{i, j}=\frac{F_{i+1, j}-F_{i-1, j}}{2 h_{x}} \hat{\boldsymbol{x}}+\frac{F_{i, j+1}-F_{i, j-1}}{2 h_{y}} \hat{\boldsymbol{y}}
$$

and the discrete Laplacian operator

$$
\nabla^{2} F_{i, j}=\frac{F_{i+1, j}-2 F_{i, j}+F_{i-1, j}}{h_{x}^{2}}+\frac{F_{i, j+1}-2 F_{i, j}+F_{i, j-1}}{h_{y}^{2}},
$$

where $h_{x}$ and $h_{y}$ are the length and width of a cell, respectively. This finite differencing of the Laplacian operator is second-order accurate, centered in space, and compact. At the $n$th time step, the discretized momentum equation appears as

$$
\frac{\boldsymbol{v}_{i, j}^{n+1}-\boldsymbol{v}_{i, j}^{n}}{\tau}+\boldsymbol{v}_{i, j}^{n} \cdot \nabla_{1} \boldsymbol{v}_{i, j}^{n}=\frac{\nu_{v}}{2} \nabla^{2}\left(\boldsymbol{v}_{i, j}^{n+1}+\boldsymbol{v}_{i, j}^{n}\right)-\nabla_{1} P_{i, j}^{n}+\boldsymbol{F}_{i, j}^{n},
$$

where $\tau$ denotes the time step. This requires the solution of the linear system of equations

$$
\left(\boldsymbol{I}-\frac{\nu_{v} \tau}{2} \nabla^{2}\right) \boldsymbol{v}_{i, j}^{n+1}=\left(\boldsymbol{I}+\frac{\nu_{v} \tau}{2} \nabla^{2}\right) \boldsymbol{v}_{i, j}^{n}+\tau \boldsymbol{S}\left(\boldsymbol{v}_{i, j}^{n}\right),
$$

i.e.,

$$
\mathcal{L}(\tau) \boldsymbol{v}_{i, j}^{n+1}=\mathcal{L}(-\tau) \boldsymbol{v}_{i, j}^{n}+\tau \boldsymbol{S}\left(\boldsymbol{v}_{i, j}^{n}\right)
$$

where $I$ denotes the identify operator,

$$
\mathcal{L}(\tau)=\boldsymbol{I}-\frac{\nu_{v} \tau}{2} \nabla^{2}
$$

and $\boldsymbol{S}\left(\boldsymbol{v}_{i, j}^{n}\right)=\boldsymbol{F}_{i, j}^{n}-\boldsymbol{v}_{i, j}^{n} \cdot \nabla_{1} \boldsymbol{v}_{i, j}^{n}-\boldsymbol{\nabla}_{1} P_{i, j}^{n}$. Equation (29) is a Helmholtz-type linear system of equations that can be solved using an iterative technique. We have chosen a multigrid method (Hackbusch, 1985; Briggs, 1987; Press et al., 1997; Tannehill et al., 1997). In order to solve (29) we need to evaluate the quantities $\boldsymbol{F}_{i, j}$ and $P_{i, j}$, as described in the following subsections.

For the concentration equation, we treat the advection part in the Lagrangian frame and the diffusion part in the Eulerian frame with a Crank-Nicholson scheme. Let $\tilde{C}$ denote the solution of the pure advection problem $\partial \tilde{C} / \partial t+\boldsymbol{v} \cdot \nabla \tilde{C}=0$, with the initial condition $\tilde{C}(\boldsymbol{x}, 0)=C_{0}$. This passive advection problem is solved using the parcel advection method (Bowman et al., 2002), which is unconditionally stable and does not require any artificial viscosity. The fluid is thought to be a collection of fluid parcels (see Figure 4). A parcel of fluid is advected with its velocity for a single time step. It then contributes to changes in the velocity and other physical properties of the surrounding fluid parcels.

We can combine advection and diffusion in the following manner:

$$
\frac{C_{i, j}^{n+1}-\tilde{C}_{i, j}^{n}}{\tau}=\frac{\nu_{c}}{2} \nabla^{2}\left(C_{i, j}^{n+1}+\tilde{C}_{i, j}^{n}\right) .
$$




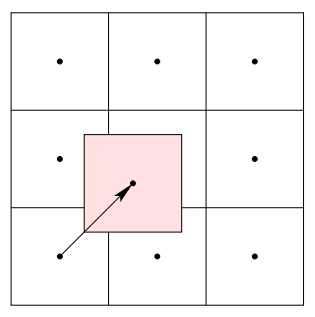

Figure 4. Advection of a parcel.

This procedure is known as operator splitting (Ames, 1977; Press et al., 1997). Equation (31) requires the solution of another linear system of partial differential equations. In a compact notation, we can rewrite the entire system of equations as $\mathcal{L}(\tau) \boldsymbol{U}^{n+1}=\mathcal{L}(-\tau) \tilde{\boldsymbol{U}}^{n}+\tau \boldsymbol{S}\left(\tilde{\boldsymbol{U}}^{n}\right)$, where

$$
\boldsymbol{U}=\left[\begin{array}{c}
v_{x} \\
v_{y} \\
C
\end{array}\right], \quad \tilde{\boldsymbol{U}}=\left[\begin{array}{c}
v_{x} \\
v_{y} \\
\tilde{C}
\end{array}\right], \quad \boldsymbol{S}=\left[\begin{array}{c}
S_{v_{x}} \\
S_{v_{y}} \\
0
\end{array}\right] .
$$

For the momentum equation, we treat both advection and diffusion in an Eulerian frame. The overall semi-implicit algorithm is summarized below:

1. Calculate the source $S$ for each equation.

2. Evaluate the quantity $\tilde{U}^{n}$.

3. Evaluate the expression $\mathcal{L}(-\tau) \tilde{\boldsymbol{U}}^{n}+\tau \boldsymbol{S}\left(\tilde{\boldsymbol{U}}^{n}\right)$.

4. Invert the operator $\mathcal{L}(\tau)$ to get $\boldsymbol{U}^{n+1}=\mathcal{L}^{-1}(\tau)\left(\mathcal{L}(-\tau) \tilde{\boldsymbol{U}}^{n}+\tau \boldsymbol{S}\left(\tilde{\boldsymbol{U}}^{n}\right)\right)$ for the next time step.

This algorithm can be used for any system of advection-diffusion equations. Thus, whenever advection dominates over diffusion, a passive tracer can be evolved primarily in a Lagrangian frame, supplemented by Eulerian diffusion.

\subsection{Computation of the Pressure Field}

The solenoidal nature of the velocity field is maintained by the pressure term in the form of the incompressibility condition. Improper treatment of the pressure field leads to a violation of the incompressibility condition, which is required for the conservation of nonlinear invariants such as energy and enstrophy. One way of avoiding such a problem is to solve first for the velocity vector potential $\boldsymbol{A}$ and then compute the velocity as $\boldsymbol{\nabla} \times \boldsymbol{A}$. Although this approach has some advantages, much of its attractiveness is lost when applied to three-dimensional flow (Tannehill et al., 1997). Moreover, there are complications in implementing the pressure boundary condition in the vector potential formulation. We have therefore developed a scheme for satisfying the incompressibility constraint directly in terms of the velocity variable. To accomplish this, the Poisson equation (21) has to be solved at every time step.

Beginning with the momentum equation, (27), in the absence of dissipation and forcing,

$$
\frac{\boldsymbol{v}_{i, j}^{n+1}-\boldsymbol{v}_{i, j}^{n}}{\tau}+\boldsymbol{v}_{i, j}^{n} \cdot \nabla_{1} \boldsymbol{v}_{i, j}^{n}=-\nabla_{1} P_{i, j}^{n}
$$

we apply the operator $\nabla_{1}$ defined by (25) to (32). We assume that the initial velocity field satisfies $\nabla_{1} \cdot \boldsymbol{v}_{i, j}^{n}=0$. In order for the computed velocity field $\boldsymbol{v}_{i, j}^{n+1}$ to satisfy the discretized incompressibility condition $\nabla_{1} \cdot \boldsymbol{v}_{i, j}^{n+1}=0$, we require that $\nabla_{1} \cdot\left(\boldsymbol{v}_{i, j}^{n} \cdot \boldsymbol{\nabla}_{1} \boldsymbol{v}_{i, j}^{n}\right)=-\boldsymbol{\nabla}_{1} \cdot \nabla_{1} P_{i, j}^{n}$. This suggests that the Laplacian $\nabla^{2} P$ in the Poisson equation (21) should be discretized by applying the operator $\nabla_{1} \cdot$ to $\nabla_{1} P_{i, j}$ :

$$
\nabla_{1}^{2} P_{i, j}=\frac{P_{i+2, j}-2 P_{i, j}+P_{i-2, j}}{4 h_{x}^{2}}+\frac{P_{i, j+2}-2 P_{i, j}+P_{i, j-2}}{4 h_{y}^{2}},
$$

instead of using the conventional compact finite-differencing of the Laplacian operator given by (26). Indeed, by maintaining the solenoidal nature of the velocity field at each time step, (33) can be used to develop a 
spatial discretization that exactly conserves the total kinetic energy of the fluid. In contrast, if the compact formula (26) is used for discretizing the Laplacian operator, the computed velocity field will not in general be solenoidal and the energy will not be conserved. In the pressure-correction method of Patankar (1980), the velocity and the pressure fields are computed simultaneously and one has to correct the pressure field at each time step until the incompressibility condition is satisfied. By differencing the equations consistently, we have developed a numerical scheme that does not require such a pressure correction.

The non-compact discretization given by (33) can be used to develop a numerical scheme that conserves energy (to machine precision), in the absence of forcing and dissipation, by spatially discretizing the momentum equation as

$$
\frac{\partial \boldsymbol{v}_{i, j}}{\partial t}=\boldsymbol{v}_{i, j} \times\left(\boldsymbol{\nabla}_{1} \times \boldsymbol{v}_{i, j}\right)-\nabla_{1}\left(\frac{v_{i, j}^{2}}{2}+P_{i, j}\right) .
$$

On taking the dot product of each side with $\boldsymbol{v}_{i, j}$, we obtain the energy evolution equation

$$
\frac{1}{2} \frac{\partial}{\partial t} v_{i, j}^{2}=-\boldsymbol{v}_{i, j} \cdot \nabla_{1} P_{i, j}^{\prime}=0
$$

where $P_{i, j}^{\prime}=v_{i, j}^{2} / 2+P_{i, j}$ is the solution to

$$
\boldsymbol{\nabla}_{1} \cdot\left[\boldsymbol{v}_{i, j} \times\left(\boldsymbol{\nabla}_{1} \times \boldsymbol{v}_{i, j}\right)\right]=\nabla_{1}^{2} P_{i, j}^{\prime} .
$$

Using the summation by parts formula

$$
\sum_{i=1}^{n} f_{i}\left(g_{i+1}-g_{i-1}\right)=f_{n} g_{n+1}+f_{n+1} g_{n}-f_{0} g_{1}-f_{1} g_{0}-\sum_{i=1}^{n}\left(f_{i+1}-f_{i-1}\right) g_{i},
$$

we then find that the total energy $\frac{1}{2} \sum_{i, j} v_{i, j}^{2} h_{x} h_{y}$ evolves according to

$$
\frac{1}{2} \frac{\partial}{\partial t} \sum_{i=1}^{n_{x}} \sum_{j=1}^{n_{y}} v_{i, j}^{2} h_{x} h_{y}=\sum_{i=1}^{n_{x}} \sum_{j=1}^{n_{y}} P_{i, j}^{\prime} \nabla_{1} \cdot \boldsymbol{v}_{i, j} h_{x} h_{y}=0,
$$

provided that $\boldsymbol{v}_{i, j} \cdot \hat{\boldsymbol{x}}$ vanishes on the boundary layers $i=0, i=1, i=n_{x}$, and $i=n_{x}+1$ (or is periodic in $i$ with period $n_{x}$ ) and $\boldsymbol{v}_{i, j} \cdot \hat{\boldsymbol{y}}$ vanishes on the boundary layers $j=0, j=1, j=n_{y}$, and $j=n_{y}+1$ (or is periodic in $j$ with period $n_{y}$ ). We thus see that the discretized equations (34) and (36) respect the energy conservation property of the advective nonlinearity.

\section{Numerical Code}

The numerical code developed throughout this study has been tested to simulate pressure-driven flow and electro-osmotic flow. A wide variety of experimental results as well as analytical results of arbitrarily simplified models exist in this case. We compare the flow characteristics computed by the numerical code with analytical and experimental results. The numerical results are found to be in good qualitative agreement with theoretical and experimental observations.

\subsection{Simulation Parameters}

Here, we describe various scales and evaluate all of the non-dimensional parameters that are defined earlier in Section 2.3. These parameters depend on various scales. The non-dimensional parameters have been evaluated from characteristic length and velocity scales based on typical laboratory experiments (Harrison et al., 1992; Crabtree et al., 2001). We summarize in Table 1 the characteristic scales and the dimensional parameters used in our numerical experiments. For the above parameters, the Debye layer thickness is about equal to the grid scale $h_{y}$. The constant $\epsilon$ is a material property of the buffer solution. For all of our numerical experiments, we use $\epsilon \sim 7.4 \times 10^{-11} \mathrm{C}^{2} /\left(\mathrm{Nm}^{2}\right)$. The values of the dimensionless parameters defined in Section 2.3 are

$$
\nu_{v}=20, \quad \nu_{c}=2 \times 10^{-3}, \quad \alpha=1.27 \times 10^{5}, \quad \kappa^{2}=4.28 \times 10^{3}, \quad \mu=0.28 .
$$


Table 1. Numerical simulation parameters.

\begin{tabular}{|l|l|l|}
\hline Length scale (channel width) & $h$ & $5 \times 10^{-5} \mathrm{~m}$ \\
Velocity scale & $U$ & $10^{-3} \mathrm{~m} / \mathrm{s}$ \\
Time scale & $h / U$ & $0.05 \mathrm{~s}$ \\
Pressure scale & $\rho U^{2}$ & $10^{-3} \mathrm{~Pa}$ \\
Potential scale & $\Phi_{*}$ & $1 \mathrm{~V}$ \\
\hline Channel length & $H$ & $80 h=4 \times 10^{-3} \mathrm{~m}$ \\
Applied input voltage & $\Phi_{H}$ & $280 \mathrm{~V}$ \\
Applied pressure at the input & $P_{0}$ & $480 \times \rho U^{2}=0.48 \mathrm{~Pa}$ \\
Kinematic viscosity of the buffer solution & $\nu$ & $10^{-6} \mathrm{~m}^{2} / \mathrm{s}$ \\
Diffusion coefficient & $D$ & $10^{-10} \mathrm{~m}^{2} / \mathrm{s}$ \\
Electrophoretic mobility & $\tilde{\mu}$ & $1.4 \times 10^{-8} \mathrm{~m}^{2} /(\mathrm{Vs})$ \\
Wall potential & $\zeta$ & $0.1 \mathrm{~V}$ \\
Boltzmann constant & $k$ & $1.38 \times 10^{-23} \mathrm{~J} / \mathrm{K}$ \\
Elementary charge & $e$ & $1.6 \times 10^{-19} \mathrm{C}$ \\
Temperature & $T$ & $300 \mathrm{~K}$ \\
Permittivity of the buffer solution & $\epsilon$ & $7.4 \times 10^{-11} \mathrm{C}^{2} /\left(\mathrm{Nm}^{2}\right)$ \\
Buffer ion density & $n$ & $3.4 \times 10^{-5} \mathrm{~mol}^{3}$ \\
Avagadro number & $N_{A}$ & $6.02 \times 10^{23} \mathrm{~mol}^{-1}$ \\
Debye layer thickness & $\lambda_{D}$ & $765 \mathrm{~nm}$ \\
Computational grid & $n_{x} \times n_{y}$ & $4097 \times 65$ \\
\hline
\end{tabular}

\subsection{Numerical Solvers}

\subsubsection{Pressure Poisson Solver}

The computation of the pressure field is one of the more expensive computational steps because the discrete Poisson equation

$$
\boldsymbol{\nabla}_{1} \cdot\left[\boldsymbol{F}_{i, j}+\boldsymbol{v}_{i, j} \times\left(\nabla_{1} \times \boldsymbol{v}_{i, j}\right)-\frac{1}{2} \nabla_{1}\left(\boldsymbol{v}_{i, j} \cdot \boldsymbol{v}_{i, j}\right)\right]=\nabla_{1}^{2} P_{i, j}
$$

has to be solved for $P_{i, j}$ at each time step. The pressure solver is based on a multigrid software library developed by Bowman et al. (2000). Iterations on a hierarchy of consecutively coarser and coarser grids are performed until convergence is achieved. The pressure field from the previous time step can be used as a good initial guess for the multigrid solver, so that convergence can be achieved in about ten iterations. In the absence of forcing and dissipation, the total kinetic energy should be exactly conserved. We tested this numerically, using a conservative second-order midpoint integrator to advance (34) forward 10 timesteps of 0.01 for a doubly periodic flow field $\boldsymbol{v}$ on $[0,1] \times[0,1]$ initially satisfying $\boldsymbol{v} \cdot \hat{\boldsymbol{x}}=\sin ^{2}(2 \pi x) \cos (2 \pi y)$ and $\nabla_{1} \cdot \boldsymbol{v}=0$ on a $16 \times 16$ grid.

In Figure 5, the numerically calculated energy and enstrophy are plotted and compared with the results obtained using a traditional compact differencing of the Laplacian operator. Discretization of the pressure Poisson equation using the non-compact formula conserves energy to as high an accuracy as desired, depending on the number of multigrid iterations.

\subsubsection{Crank-Nicholson Solver}

The viscous term is treated with unconditional stability at the expense of using a Crank-Nicholson method. The nonlinearity associated with the advective term in the momentum equation is treated explicitly on the right-hand side. We have developed a $\mathrm{C}^{++}$Crank-Nicholson solver around a core recursive multigrid routine. The Crank-Nicholson solver is based on an efficient multigrid solver for the discretized Helmholtz equation $\nabla^{2} u+\lambda u=f$, where $\lambda$ is a constant and $f$ is a source function. A red-black ordered Gauss-Seidel smoother is used to reduce the non-smooth component of the error in the fine grid. The divergence-free velocity computed in the previous time step is used as an initial guess. It is found that the Crank-Nicholson solver converges in about ten iterations. 


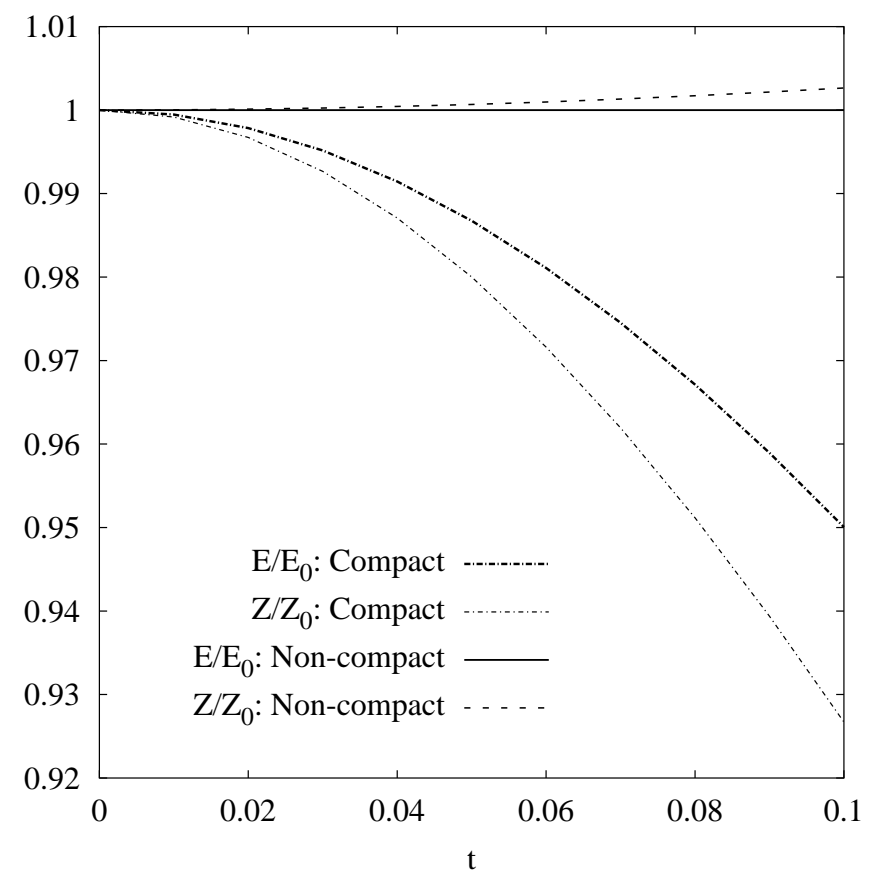

Figure 5. Scaled energy and enstrophy evolution as predicted with the compact and non-compact pressure formulations.

Many theoretical and experimental studies have been done to explain the fundamental characteristics of pressure-driven flow (Bowen et al., 1976; Rice \& Whitehead, 1965; Burgreen \& Nakache, 1964). We consider a two-dimensional pressure-driven flow in a rectangular channel of aspect ratio $80: 1$. A parabolic (Poiseuille) profile in the streamwise velocity field develops as a result of a pressure drop along the length of the channel.

\subsection{Electro-Osmotic Velocity Field}

In this section we illustrate the electrokinetic effect in two-dimensional electro-osmotic flow in the absence of an applied pressure drop. The Debye layer thickness $\lambda_{D}$, which depends on the bulk ionic concentration of the liquid inside the channel and plays a key role in producing electro-osmotic flow, is typically small compared with the width $h$ of the channel. A decrease of bulk concentration will increase the Debye thickness and decrease the dimensionless parameter $\kappa=h / \lambda_{D}$. Since the Debye layer establishes the charge separation that leads to electro-osmotic flow (see Section 1), a change in $\kappa$ will affect the electro-osmotic velocity profile. This effect was studied analytically by Rice \& Whitehead (1965) using a one-dimensional mathematical model. We present in Figure 6 the electro-osmotic velocity profile for different values of effective ion density $n$ for a two-dimensional channel. For large values of $n$ (and hence $\kappa$ ) we see that the velocity has a flat profile (and $\psi$ is negligible) across the channel, except in a narrow region near the wall. This is the expected electro-osmotic velocity profile. As $n$ becomes smaller, departure from electro-osmotic flow becomes noticeable. When the Debye thickness is comparable with the characteristic length scale $h$, the internal potential $\psi$ is non-zero far away from the walls. For low values of $n$, the velocity profile takes on a parabolic form, due to the overlapping of the Debye layer with the entire cross section.

\subsection{Evolution of the Concentration Field}

Numerical experiments have been performed to study injection of a sample under the influence of a constant electric field and pressure drop. The normalized sample concentration is set equal to one on a strip at the input boundary and to zero elsewhere in the computational domain. One aim of the simulations was to 


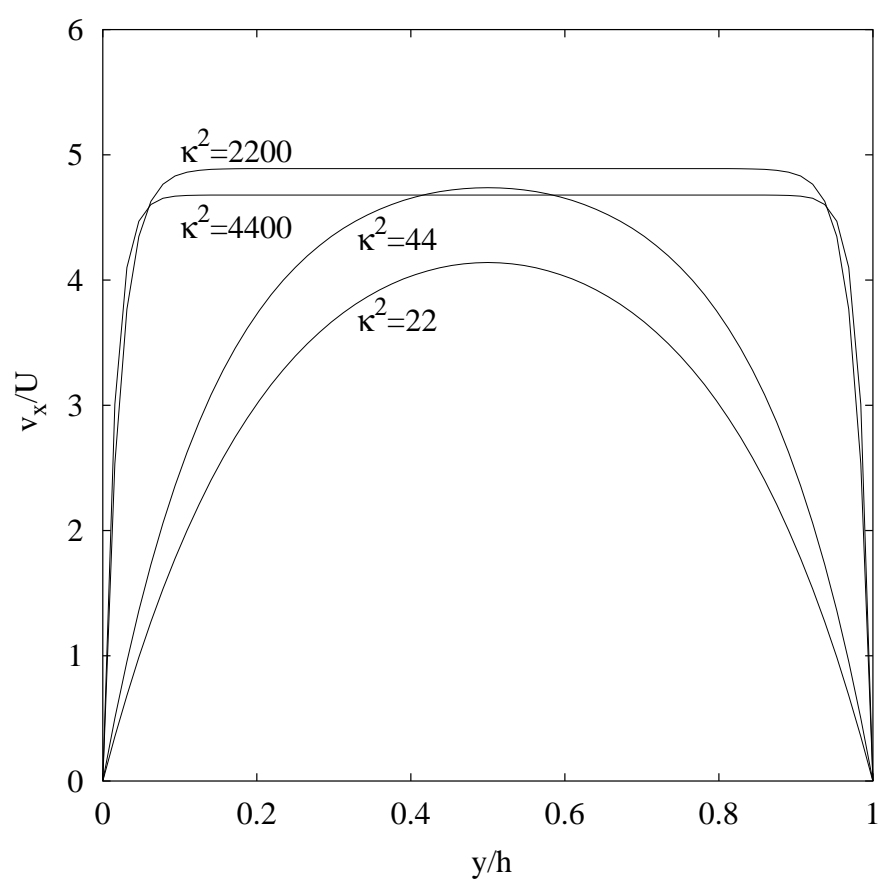

Figure 6. Electro-osmotic velocity profiles for different values of $\kappa^{2}$.

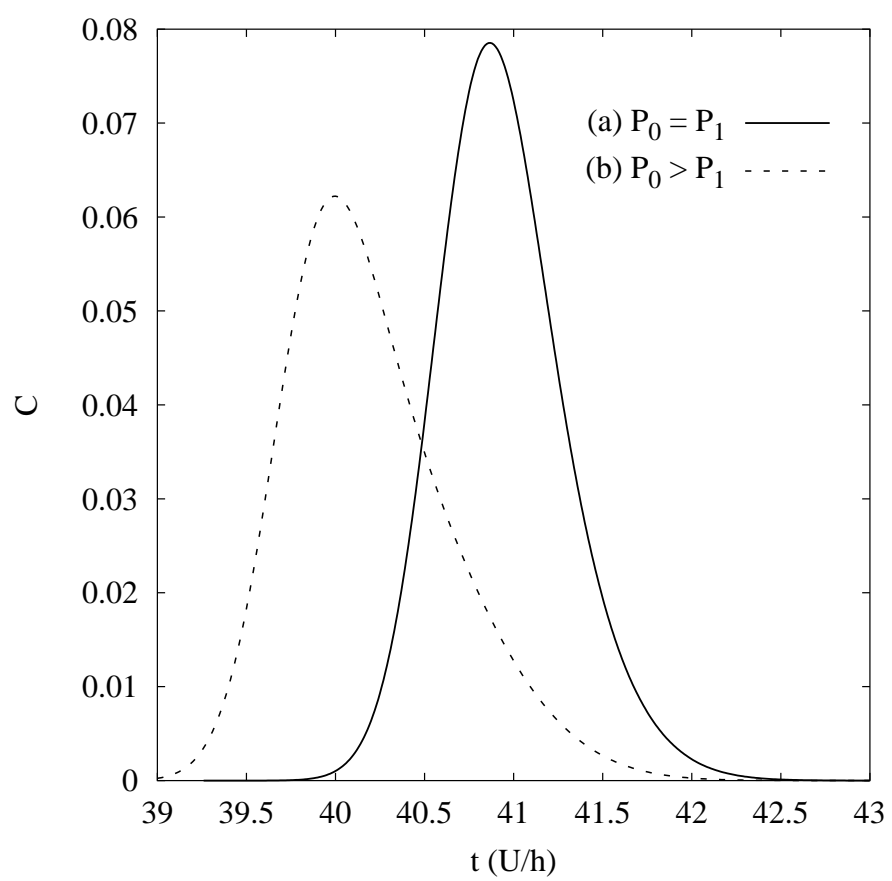

Figure 7. Distribution of $C$ along the center line of the channel at a distance $3 H / 4$ downstream from the input boundary. 
(a)

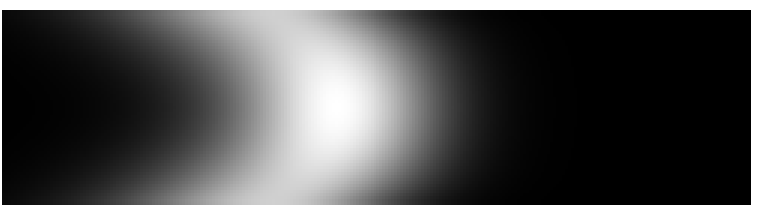

(b)

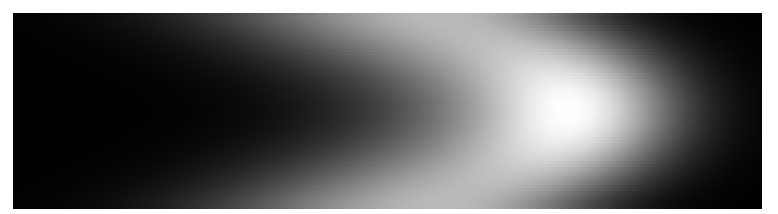

Figure 8. Distribution of $C$ at $t=44$ with (a) only an applied potential and (b) applied potential and pressure drops.

study the concentration distribution if the sample is injected with a constant electric field in the absence of a pressure drop between the input and output boundaries, so that the sample travels toward the output boundary solely due to the applied electromotive force. Even when the pressure drop across the channel is zero, one cannot assume that $P=0$ in the interior of the channel; a pressure field is still needed to maintain the incompressibility of the fluid. Therefore, in contrast to the practice in some previous studies (Patankar \& Hu, 1998), the $\nabla P$ term in the momentum equation should not be neglected. Another aim of the simulations was to see the effect of a small pressure gradient on the concentration distribution. The evolution of the concentration field is the result of two major mechanisms: advection (i.e., electro-osmosis and electrophoresis) and diffusion. Because of the low value $\left(\sim 10^{-3}\right)$ of the non-dimensional parameter $\nu_{c}=D /(U h)$, transport of the sample is dominated by advection.

We simulated a rectangular sample plug near the input boundary of a channel with aspect ratio $80: 1$. The computational resolution $4097 \times 65$ was chosen to be sufficient to resolve the Debye layer. To study the effect of dispersion, which becomes important in a long simulation, we report the results of two numerical experiments: flow due to an applied potential drop but (a) without any pressure drop and (b) with a pressure drop. The profile of $C$, which is usually called an electropherogram, is calculated at a point $3 H / 4$ downstream from the input boundary and depicted in Figure 7. The distribution of $C$ for both cases is illustrated in Figure 8. The electropherogram is nearly symmetric in $x$ if there is no pressure drop between the input and output boundaries. The sample concentration is expected to have a Gaussian distribution when injected by an electro-osmotic flow because the velocity profile is flat except in the Debye region. Note that the electropherogram for case (a) is not perfectly symmetric, because of the enhanced value of $\lambda_{D}$ that we used.

In contrast, in the presence of a small pressure gradient, equivalent to a pressure drop of $2 \mathrm{~mm}$ of water across the device (which can readily occur in an experiment), the distribution of $C$ is broader and markedly asymmetric. We see that a pressure drop can cause dispersion, which is undesirable in electrophoretic species separation. In our simulation, the sample has traveled only a physical distance of about 3 mm. However, we expect to find a qualitatively similar dispersive effect, as seen in experiments (Crabtree et al., 2001), if the same pressure drop is distributed over a greater distance since in each case the same amount of work will be done by the $\nabla P$ force on the fluid over the length of the channel.

\section{Conclusions}

We have presented a numerical model of electro-osmosis that couples passive scalar advection with Eulerian diffusion. This model consists of a set of elliptic, parabolic and hyperbolic partial differential equations. A principle contribution of this work is the development of a semi-implicit multigrid algorithm for the incompressible Navier-Stokes equation, which uses a non-compact discretization of the Poisson equation for the pressure field to obtain a divergence-free velocity. This second-order center-differenced algorithm 
was designed to conserve energy in the inviscid limit. The implications of the failure of many numerical algorithms to respect fundamental inviscid invariants (even if the actual system is dissipative) is discussed further in de Frutos \& Sanz-Serna (1994), Bowman et al. (1997), Shadwick et al. (1999), and Kotovych \& Bowman (2002).

$\mathrm{A} \mathrm{C}^{++}$multigrid Crank-Nicholson solver is used to handle the viscous terms, which for the velocity equations are dominant. This solver converges rapidly; the number of iterations is roughly independent of the number of grid points. Once the velocity field is updated via the Navier-Stokes equation, the concentration equation is solved in Lagrangian coordinates; although an Eulerian upwind scheme can provide a stable numerical scheme for passive advection, it introduces too much numerical diffusion and does not guarantee that the concentration field remains everywhere non-negative. Instead, the fluid is modeled as a collection of parcels, each of which has a mean velocity, concentration, and position. The momentum equation is solved on an Eulerian grid and the fluid body force is evaluated by solving the appropriate Laplace and Helmholtz equations. The pressure is calculated at the cell centroid by solving a Poisson equation. At each time step, a parcel is assigned a new velocity equal to the area-weighted average of the fluid velocities in the cells it overlaps. In a similar manner, parcels distribute their concentration to the cells they overlap.

Alternative numerical advection algorithms can be found in Holt (1984); LeVeque (1990); Tannehill et al. (1997). Another alternate is the flux-corrected transport algorithm of Boris \& Book (1964), which is an Eulerian method based on Lagrangian considerations. Although this method can ensure positivity of the transported quantity and has been tested for several applications (Boris \& Book, 1976), numerical diffusion necessitates the use of an anti-diffusion stage (Boris \& Book, 1964), which must be tailored to the particular problem. However, if there is a sharp gradient in the solution, Eulerian numerical methods have difficulties (Woodward \& Colella, 1984; LeVeque, 1990). We find that our parcel advection algorithm predicts the solution of an advection equation very well, even when a sharp gradient exists in the solution, as is typically the case in electrophoretic separation.

Although our algorithm should have broader applications to many problems involving the passive advection of a scalar field, we applied it in this work to verify numerically the experimentally observed dispersive broadening of a concentration pulse due to a pressure gradient (Crabtree et al., 2001). In the case of electro-osmotic flow, our simulations confirm that pressure effects can in principle halt, retard or even reverse the effects of electro-osmotic pumping. Perhaps more attention should be given to this pressure effect in developing future electrophoretic technologies.

\section{Acknowledgments}

The authors thank Christopher J. Backhouse of the Department of Electrical and Computer Engineering, University of Alberta and H. John Crabtree of Micralyne Inc. for valuable discussions and helpful advice.

\section{References}

Alam, M. J. (2000). A fully Lagrangian advection scheme for electro-osmotic flow. Master's thesis, University of Alberta, Edmonton, Alberta, Canada.

Ames, W. F. (1977). Numerical Methods for Partial Differential Equations. Academic Press, San Diego, California.

Andreev, V. P., and Lisin, E. E. (1993). On the mathematical-model of capillary electrophoresis. Chromatograhia, 37, $202-210$.

Arulanandam, S., and Li, D. Q. (2000). Determining zeta potential and surface conductance by monitoring the current in electro-osmotic flow. Journal of Colloid and Interface Science, 225, 421-428.

Babskii, V. G., Zhukov, M. Y., and Yudovich, V. I. (1983). Mathematical Theory of Electrophoresis. Consultants Bureau, New York.

Boris, J., and Book, D. (1964). Flux-corrected transport I. SHASTA, a fluid transport algorithm that works. Journal of Computational Physics, 11, 38-69.

Boris, J., and Book, D. (1976). Solution of continuity equations by the method of flux-corrected transport. Methods in Computational Physics, 16, 85-129.

Bowen, B. D., Levine, S., and Epstein, N. (1976). Fine particle deposition in laminar flow through parallel-plate and cylindrical channels. Journal of Colloid and Interface Science, 54, 375-390.

Bowman, J. C., Shadwick, B. A., and Morrison, P. J. (1997). Exactly conservative integrators. In 15th IMACS World Congress on Scientific Computation, Modelling and Applied Mathematics pp. 595-600. Berlin Wissenschaft \& Technik. 
Bowman, J. C., Zeiler, A., and Biskamp, D. (2000). A multigrid algorithm for nonlocal collisional electrostatic drift-wave turbulence. Journal of Computational Physics, 158, 239-261.

Bowman, J. C., Wasylishen, S. J., and Alam, M. J. (2002). Parcel advection: A fully Lagrangian advection scheme. To be submitted to J. Computational Physics.

Briggs, W. I. (1987). A multigrid tutorial. SIAM, Pennsylvania.

Burgreen, D., and Nakache, F. R. (1964). Electrokinetic flow in ultrafine capillary. J. Phys. Chem., 68, 1084-1091.

Courant, R., Isaacson, E., and Rees, M. (1952). On the solution of nonlinear hyperbolic differential equations by finite difference. Comm. Pure Appl. Math., 5, 243-255.

Crabtree, H. J., Cheong, E. C. S., Tilroe, D. A., and Backhouse, C. J. (2001). Microchip injection and separation anomalies due to pressure effects. Anal. Chem., 73, 4079-4086.

Culbertson, C. T., and Jorgenson, J. W. (1994). Flow counterbalanced capillary electrophoresis. Anal. Chem., 66, 955-962.

Culbertson, C. T., Jacobson, S. C., and Ramsey, J. M. (1998). Dispersion sources for compact geometries on microchips. Anal. Chem., 70, 3781-3789.

de Frutos, J., and Sanz-Serna, J. M. (1994). Erring and being conservative. In Numerical Analysis 1993, edited by Griffiths, D. F., and Watson, G. A. Pitman Research Notes in Mathematics, pp. 75-88. Longmans Scientific and Technical, Harlow.

Debye, P., and Hückel, E. (1923). Zur Theorie der Elektrolyte. Physik. Zeitschr., 24, 185-206.

Ermakov, S. V., Jacobson, S. C., and Ramsey, J. M. (1998). Computer simulations of electrokinetic transport in microfabricated channel structures. Anal. Chem., 70, 4494-4504.

Ermakov, S. V., Jacobson, S. C., and Ramsey, J. M. (2000). Computer simulations of electrokinetic injection techniques in microfluidic devices. Anal. Chem., 15, 3512-3517.

Fletcher, P., Haswell, S., and Paunov, V. (1999). Theoritical considerations of chemical reactions in micro-reactors operating under electro-osmotic and electrophoretic control. Analyst, 124, 1273-1282.

Hackbusch, W. (1985). Multigrid methods and applications. Springer, New York.

Harrison, D. J., Manz, A., Fan, Z., Lüdi, H., and Widmer, H. M. (1992). Capillary electrophoresis and sample injection systems integrated on a planar glass chip. Anal. Chem., 64, 1926-1932.

Harrison, D. J., Glavina, P. G., and Manz, A. (1993). Towards miniaturized electrophoresis and chemical analysis systems on silicon: an alternative to chemical sensors. Sens. Actuators B, B10, 107-116.

Holt, M. (1984). Numerical Methods in Fluid Dynamics. Springer, New York.

Jorgenson, J. W., and Lukacs, K. D. (1981). Zone electrophoresis in open-tubular glass capillaries. Anal. Chem., 53, 1292-1302.

Kotovych, O., and Bowman, J. C. (2002). An exactly conservative integrator for the $n$-body problem. To appear in J. Phys. A.: Math. Gen.

Leslie, D. C. (1973). Developments in the Theory of Turbulence. Clarendon Press, Oxford.

LeVeque, R. J. (1990). Numerical Methods for Conservation Laws. Birkhauser Verlag, Boston.

Levine, S., and Neale, G. H. (1976). The prediction of electrokinetic phenomena within multiparticle systems I. Electrophoresis and electro-osmosis. Journal of Colloid and Interface Science, 47, 520-529.

Morrison, F. A., JR., and Stukel, J. J. (1970). Electrophoresis of an insulating sphere normal to a conducting plane. Journal of Colloid and Interface Science, 33, 88-93.

Mosher, R. A., Saville, D. A., and Thormann, W. (1992). The Dynamics of Electrophoresis. VCH, Weinheim.

Patankar, N. A., and Hu, H. H. (1998). Numerical simulation of electro-osmotic flow. Anal. Chem, 70, 1870-1881.

Patankar, S. V. (1980). Numerical Heat Transfer and Fluid Flow. Hemisphere, Washington.

Press, W. H., Teukolsky, S. A., Vetterling, W. T., and Flannery, B. P. (1997). Numerical Recipes in C: The art of scientific computing. Cambridge University Press, Cambridge.

Reed, L. D., and Morrison, F. A., Jr. (1976). Hydrodynamic interactions in electrophoresis. Journal of Colloid and Interface Science, 54, 117-133.

Rice, C., and Whitehead, R. (1965). Electrokinetic flow in a narrow cylindrical capillary. The Journal of Physical Chemistry, 69, 4017-4024.

Richtmyer, R. D., and Morton, K. W. (1957). Difference Methods for Initial-Value Problems. Interscience Publishers, New York.

Ritchie, H. (1986). Eliminating the interpolation associated with the semi-Lagrangian scheme. American Meteorological Society, 114, 135-146.

Ruess, F. F. (1809). Memoires de la Société Imperiale de Naturalistes de Moskou. pp. 327-336.

Saville, D. A. (1977). Electrokinetic effects with small particles. Ann. Rev. Fluid Mech., 9, 321-327.

Seiler, K., Fan, Z. H., Fluri, K., and Harrison, D. J. (1994). Electroosmotic pumping and valveless control of fluid flow within a manifold of capillaries on a glass chip. Anal. Chem, 66, 3485-3491.

Shadwick, B. A., Bowman, J. C., and Morrison, P. J. (1999). Exactly conservative integrators. SIAM J. Appl. Math., 59, $1112-1133$.

Sidilkover, D., and Ascher, U. M. (1995). A multigrid solver for the steady-state navier-stokes equations using the pressure-poisson formulation. Computational and Applied Mathematics, 14, 21-35.

Tannehill, J. C., Anderson, D. A., and Pletcher, R. H. (1997). Computational Fluid Mechanics and Heat transfer. Taylor and Francis. von Helmholtz, H. L. F. (1879). Studies of electric boundary layers. Wied. Ann., 7, 337-382.

Woodward, P., and Colella, P. (1984). The numerical simulation of two-dimensional fluid flow with strong shocks. Journal of Computational Physics, 54, 115-173. 